\title{
Effect of Hawthorn Leaf Flavonoids in Dehydroepiandrosterone-Induced Polycystic Ovary Syndrome in Rats
}

\author{
Yanhua Shi $^{a}$ Xiang Kong ${ }^{b}$ Hongmei Yin ${ }^{b}$ Wentong Zhang ${ }^{b}$ Wei Wang ${ }^{b}$ \\ a Jinan Second People's Hospital, Jinan City, PR China; ${ }^{b}$ Department of Obstetrics and Gynecology, Shengli Oilfield \\ Central Hospital, Dongying City, PR China
}

\section{Keywords}

Hawthorn leaf flavonoids · Polycystic ovary syndrome ·

Dehydroepiandrosterone $\cdot$ Insulin resistance $\cdot$ Hormonal antioxidant

\section{Abstract \\ Objective: To evaluate the potential beneficial effects of hawthorn leaf flavonoids (HLF) against polycystic ovary syn- drome (PCOS) in a rat model of disease and elucidate the underlying molecular mechanism. Methods: The PCOS model was established by subcutaneous injection of dehy- droepiandrosterone (DHEA, $60 \mathrm{mg} / \mathrm{kg} /$ day) for 21 consecu- tive days. HLF (200 mg/kg/day) were orally administered si- multaneously or after the injection. The body weight was regularly monitored and recorded. The ovaries were weighed and histologically examined via hematoxylin and eosin staining. The number of follicular cysts was counted under a light microscope. The serous hormones were measured us- ing enzyme-linked immunosorbent assay kits. Insulin resis- tance (IR) was calculated as HOMA-IR = fasting insulin $(\mu \mathrm{U} / \mathrm{L})$ $\times$ fasting glucose $(\mathrm{mm}) / 22.5$. The estrous cycle was deter- mined by vaginal smear. The relative expression of tumor necrosis factor-a and interleukin- 6 was measured by real-}

time polymerase chain reaction. The superoxide dismutase activity and malondialdehyde content was determined using commercially available kits. Results: DHEA induced a significant increase of body weight, ovary weight, number of follicular cysts, serous hormones, IR, inflammatory cytokines, and oxidative stress, and it also impaired the estrous cycle. Oral administration of HLF greatly alleviated these complications. Little toxicity of HLF was observed in our rat model. Conclusion: HLF manifest protective effects against PCOS progression in the animal model, which may hold great promise for future clinical applications.

(C) 2018 S. Karger AG, Basel

\section{Introduction}

Polycystic ovary syndrome (PCOS) is one of the most common and complicated reproductive disorders in women [1]. It features hyperandrogenism, polycystic ovaries, and anovulation [2]. It is estimated to affect $5-10 \%$ of women between the ages of 18 and 44 years [3]. Epidemiological investigation has suggested that both en-

Yanhua Shi and Xiang Kong contributed equally to this work.

\section{KARGER}

(c) 2018 S. Karger AG, Basel 
vironmental and genetic factors contribute to the etiology of this disease [4]. The diagnostic standard relies on the following 3 clinical signs: no ovulation, high androgen levels, and the appearance of multiple small cysts [5]. The clinical manifestations of PCOS include menstrual disorders, infertility, high levels of masculinizing hormones, and metabolic syndrome associated with hyperinsulinemia, diabetes mellitus, and endometrial cancer. The pathogenesis of PCOS is frequently associated with excessive production of androgenic hormones in the ovaries by either an excessive amount luteinizing hormone (LH) from the anterior pituitary gland or a high level of insulin in the blood, alone or in combination [6]. The majority of women with PCOS suffer from insulin resistance (IR) and/or obesity [7]. In addition, recent evidence suggests that PCOS may be associated with chronic inflammation, where the inflammatory cytokines show a positively correlation with anovulation and other PCOS symptoms [8].

Most PCOS patients require prolonged clinical management, including lifestyle adjustments and medication. The primary and practical goals of treatments are: improvement of IR, restoration of fertility, control of hirsutism and acne, restoration of regular menstruation, and prevention of endometrial hyperplasia and malignant cancer [9]. Since an intimate association between PCOS and overweight or obesity was established, body weight loss is proven to be the most effective method to restore normal ovulation and menstruation [10]. The mainstay medications for the clinical treatment of PCOS include oral contraceptives and metformin. The former increases sex hormone-binding protein, which in turn reduces free testosterone and mitigates PCOS symptoms. Metformin is a drug commonly used in type 2 diabetes to reduce IR; in many cases, this supports ovarian function and maintains normal ovulation [11]. Despite the well-recognized therapeutic value of metformin, reports have emerged disclosing potential side effects, including an increased risk of ectopic pregnancy and congenital malformations [12]. In this regard, complementary and alternative therapeutic strategies need to be evaluated to alleviate PCOS.

It has been discovered that flavonoid compounds extracted from natural plants have high therapeutic value for a variety of human diseases. Hawthorn leaf flavonoids (HLF) isolated from the hawthorn leaf have been reported to possess a broad spectrum of medicinal properties. For example, they have been shown to alleviate nonalcoholic liver disease by enhancing the adiponectin/AMPactivated protein kinase pathway [13]. Fu et al. [14] reported that HLF decreased inflammation related to acute myocardial ischemia/reperfusion in anesthetized dogs.
The antioxidant and anti-inflammatory effects of HLF have also been shown to protect against diabetes-induced cardiomyopathy in rats via the protein kinase $\mathrm{C}(\mathrm{PKC})-\alpha$ signaling pathway [15]. In view of the critical role of inflammation milieu in the etiology of PCOS, we sought to evaluate the potential therapeutic benefit of HLF in PCOS by employing a rat model of DHEA-induced disease. Our study, for the first time, highlights the potential clinical application of HLF in PCOS patients.

\section{Materials and Methods}

\section{Animals}

Sprague-Dawley rats were purchased from the Vitalriver (Beijing, China) and kept at a constant temperature of $25 \pm 2{ }^{\circ} \mathrm{C}$ with a 12-h light-dark cycle. The animals had free access to drinking water and food ad libitum and were acclimated for 1 week before the experiments. The protocol involving animal-related studies was reviewed and approved by Shengli Oilfield Central Hospital.

\section{PCOS Rat Model Establishment and HLF Treatment}

In total, 49 female rats were used in studies. On the one hand, 29 prepubertal female rats (23 days old) were randomly divided into 4 experimental groups: control (subcutaneous [s.c.] injection of saline, $n=8$ ); dehydroepiandrosterone (DHEA; s.c. injection of DHEA, $60 \mathrm{mg} / \mathrm{kg} /$ day, $n=8$ ); DHEA + HLF (s.c. injection of DHEA, $60 \mathrm{mg} / \mathrm{kg} /$ day, plus oral administration of HLF, $200 \mathrm{mg} /$ $\mathrm{kg} / \mathrm{day}, n=7$ ); and HLF (oral administration of HLF, $200 \mathrm{mg} / \mathrm{kg}$ / day, $n=6$ ) groups. The PCOS model was established following a previously described protocol. Briefly, DHEA rats were injected s.c. with DHEA dissolved in $0.2 \mathrm{~mL}$ sesame oil for 21 consecutive days. HLF were orally administered simultaneously with $200 \mathrm{mg} /$ $\mathrm{kg} /$ day referred to in the previous report.

On the other hand, 18 female rats (53 days old) were used for the administration of HLF after the PCOS rats were established: control (s.c. injection of saline, $n=6$ ); DHEA (the successful PCOS model rats received saline orally, $n=7$ ); and DHEA + HLF (the successful PCOS model rats received HLF orally, $200 \mathrm{mg} / \mathrm{kg} / \mathrm{day}$, $n=7$ ) groups. All 3 groups were treated for 3 weeks.

\section{Body Weight and Vaginal Smears}

Body weight was monitored daily during the whole experimental procedure. The estrous cycle was determined by microscopic examination of cell types on vaginal smears obtained regularly from day 11 after the DHEA treatment. Measurements lasted until the rats were humanely sacrificed.

\section{Measurement of Serous Glucose, Insulin, Estradiol, and}

Progesterone Levels

After the experiments and fasting for $12 \mathrm{~h}$, euthanasia was performed with an overdose of sodium pentobarbital. The blood samples were collected, and serous glucose, insulin, estradiol, and progesterone levels were determined. The serous concentration of glucose was determined using 7600-210 AutoAnalyzer (Hitachi, Tokyo, Japan). The serum insulin level was measured using an ultrasensitive rat insulin ELISA kit (Mercodia, Uppsala, Sweden) by following the manufacturer's manual. IR (homeostatic model 
assessment [HOMA]-IR) was calculated by the formula: HOMA$\mathrm{IR}=$ fasting insulin $(\mu \mathrm{U} / \mathrm{L}) \times$ fasting glucose $(\mathrm{mM}) / 22.5$. The concentrations of serum estradiol and progesterone were determined using electrochemiluminescence immunoassay (Roche Diagnostics, Mannheim, Germany) according to the provider's instruction.

\section{Histological Examination of the Ovaries}

The ovaries were resected and weighed, and immediately fixed in $4 \%$ paraformaldehyde overnight at $4^{\circ} \mathrm{C}$. After washing with tap water, they were embedded in paraffin and cut into 5-mm-thick sections. These sections were then deparaffinized with xylene, rehydrated with graded ethanol, and stained with hematoxylin and eosin (H\&E). Images were acquired using a DP-70 digital light microscope system (Olympus, Tokyo, Japan). The number of follicular cysts was counted in 3 random fields per sample.

\section{Real-Time Polymerase Chain Reaction}

Total RNA was extracted from ovary tissues with Trizol reagent following the manufacturer's instructions. The integrity of RNA was quality-checked by BioAnalyzer 2100 (Agilent, CA, USA). The concentration of RNA was determined by NanoDrop 2000 (ThermoFisher, MA, USA). The cDNA was prepared using the highcapacity cDNA reverse transcription kit (ThermoFisher) following the manufacturer's guide. Real-time PCR was performed in triplicate using a SYBR Green PCR Master Mix (Applied Biosystems, NJ, USA). The relative expressions of tumor necrosis factor (TNF)- $\alpha$ and interleukin (IL)- 6 were calculated using the $2^{-\Delta \Delta C t}$ method, and normalized to GAPDH. The primer sequences were available upon request.

Superoxide Dismutase Activity and Malondialdehyde Content

The superoxide dismutase (SOD) activity in the indicated ovary tissues was determined using the total (T)-SOD assay kit (A001$1-1$, Jiancheng Bioengineering Institute, Nanjing, China) in accordance with the manufacturer's instruction. The malondialdehyde (MDA) content of the ovaries was measured with the MDA assay kit (A003-1, Jiancheng Bioengineering Institute). The absorption was recorded on the SpectraMax iD5 multimode microplate reader (Molecular Devices, CA, USA).

\section{Statistical Analysis}

Data were obtained from at least 3 independent experiments. All results were expressed as mean \pm SD. Data were analyzed and processed by GraphPad Prism v7.0 software. The Student $t$ test was employed for pairwise comparison. One-way analysis of variance (ANOVA), followed by the Tukey test, was applied for multiple comparisons. The $\chi^{2}$ test was for the analysis of the number of estrous cycles in each group. $p<0.05$ was considered a significant difference.

\section{Results}

\section{Establishment of PCOS Rat Model}

The method for establishment of PCOS rat model referred to the protocol described by Jang et al. [16]. The experimental schema is illustrated in Figure 1. The dosage of

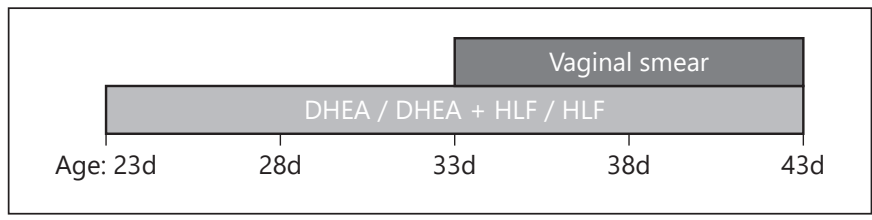

Fig. 1. Experimental protocols for PCOS model and HLF treatment. The rats were treated with DHEA and/or HLF for 21 days, i.e., from day 23 to day 43 (23d-43d). Vaginal smears were obtained daily, beginning on day 33 after birth.

HLF used in this study was consistent with a previous report [15]. Body weight and estrous cycle were monitored from day 11 after s.c. injection of DHEA. For the following experiment, the test of vaginal exfoliated cytology was taken for 10 days and successful PCOS rats were used.

\section{Effect of HLF on the Body Weight in PCOS Rats}

We first evaluated the impact of HLF administration on the body weight of the recipient rats. As shown in Figure $2 \mathrm{a}$, the growth curve in the PCOS group was significantly higher after DHEA treatment for 11 days in comparison with either control or HLF rats, which indicated the successful induction of PCOS in the DHEA group. Oral administration of HLF inhibited the weight gain in the PCOS rats, suggesting that HLF potentially alleviated disease progression. The possible toxicity of HLF at our dosage was excluded by monitoring the body weight in rats receiving oral HLF alone, where no difference was observed when compared to controls. We further statistically compared the body weight of the 4 groups of rats on days 33, 38, and 43 (Fig. 2b). The body weight in DHEA rats was remarkably higher than in controls and HLFtreated PCOS rats (day 33: $91.3 \pm 1.2 \mathrm{~g}$ in controls vs. $104.2 \pm 2.1 \mathrm{~g}$ in DHEA vs. $107.1 \pm 1.4 \mathrm{~g}$ in DHEA + HLF vs. $91.4 \pm 1.5 \mathrm{~g}$ in HLF; day 38: $122.3 \pm 1.5 \mathrm{~g}$ in controls vs. $136.5 \pm 2.8 \mathrm{~g}$ in DHEA vs. $127.1 \pm 1.3 \mathrm{~g}$ in DHEA + HLF vs. $119.4 \pm 1.8 \mathrm{~g}$ in HLF; day $43: 139.3 \pm 2.2 \mathrm{~g}$ in controls vs. $150.2 \pm 2.1 \mathrm{~g}$ in DHEA vs. $145.1 \pm 1.4 \mathrm{~g}$ in DHEA + HLF vs. $137.8 \pm 1.4 \mathrm{~g}$ in HLF).

The administration of HLF after the establishment of PCOS rats (online suppl. Figure S1A; for all online suppl. material, see ww.karger.com/doi/10.1159/000493895) also decreased the body weight compared to in the model $(305.6 \pm 10.3 \mathrm{~g}$ in DHEA vs. $270.8 \pm 10.1 \mathrm{~g}$ in DHEA + HLF, $p<0.05)$. Our data clearly demonstrate that we succeeded in establishing PCOS in DHEA-treated rats, and that co- or postadministration of HLF significantly prevented disease progression. 


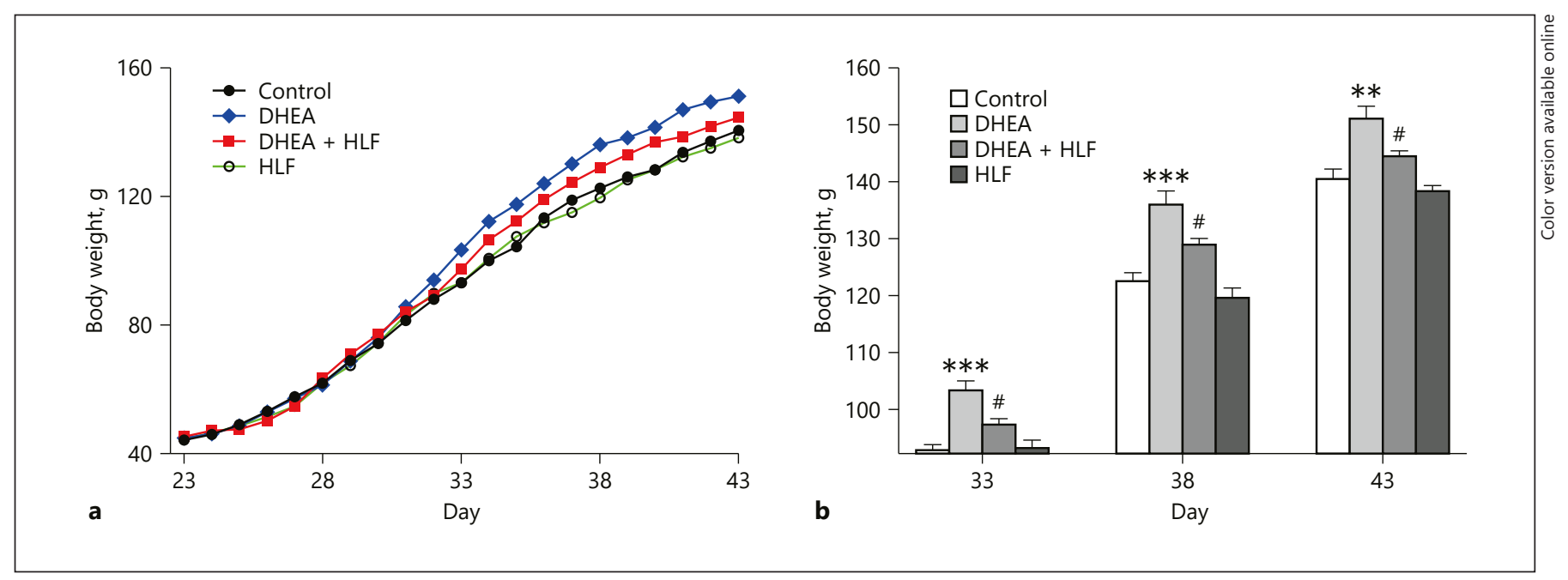

Fig. 2. Effect of HLF on the body weight in rats. a Growth curves of each group. Values represent the mean. b Body weight on days 33, 38, and 43 after birth. Values represent the mean \pm SEM. ${ }^{* *} p<0.01,{ }^{* * *} p<0.001$ control versus DHEA group, ${ }^{\#} p<0.05$ DHEA versus DHEA + HLF group. One-way ANOVA, Tukey post hoc test.

Effect of HLF on the Weight and Morphology of the

Ovaries in Rats

Next, we examined the weight of the ovaries of the sacrificed rats. The weight in the DHEA groups were significantly higher than in controls $(155.3 \pm 18.1$ vs. $103.3 \pm$ $16.8 \mathrm{mg}, p<0.05$, Fig. $3 \mathrm{a} ; 197.3 \pm 10.5$ vs. $150.5 \pm 12.1 \mathrm{mg}$, $p<0.05$, online suppl. Fig. S1B). Administration of HLF simultaneously or after establishing PCOS completely restored the organ weight back to that of the controls (103.3 \pm 16.8 vs. $95.3 \pm 15.2 \mathrm{mg}, p>0.05$, Fig. $3 \mathrm{a} ; 165.7 \pm 9.2$ vs. $150.5 \pm 12.1 \mathrm{mg}, p>0.05$, online suppl. Fig. S1B). HLF uptake had no impact on ovarian weight in comparison with the controls. The appearance of ovarian follicular cysts represented the pivotal clinical characteristic during PCOS progression.

We then cautiously examined the morphology of the ovaries from each group of rats. Histological sections were subjected to $\mathrm{H} \& \mathrm{E}$ staining and then inspected by experienced pathologists. The number of follicular cysts was counted under a light microscope across the transection. As shown in Figure 3b, coadministration of HLF markedly decreased the occurrence of follicular cysts (7.1 \pm 0.8 vs. $4.2 \pm 0.8$ per section, $p<0.05)$, as did the postadministration of HLF ( $5.7 \pm 0.5$ vs. $3.7 \pm 0.6$ per section, $p<0.05$, online suppl. Fig. S1C). This indicates the protective effect of HLF. Representative images of $\mathrm{H} \& \mathrm{E}$ staining are in Figure $3 \mathrm{c}$ and online supplementary Figure S1E. Ovaries from controls and HLF rats exhibited a nor-

HLF Manifest Protective Effects against PCOS mal appearance, with an outward shape, a central medulla composed of fibromuscular stroma and a number of blood vessels, a peripheral cortex containing abundant follicles, and a large corpora lutea. In sharp contrast, ovaries from PCOS rats had a swollen appearance, multiple dilated cystic follicles, atretic follicles, and well-developed theca interna cells, supporting fully developed PCOS. Intriguingly, both the size and number of follicular cysts were decreased in the HLF-treated rats, unambiguously indicating that coadministration of HLF inhibits the development of follicular cysts.

\section{Effect of HLF on the Levels of Serum Hormones in Rats}

Next, we evaluated steroidogenic function in the DHEA-induced PCOS model. The serous levels of estradiol and progesterone were determined using commercially available kits. As shown in Table 1 , the serum content of luteinizing hormone, follicle-stimulating hormone, estradiol, progesterone, and testosterone was significantly increased in the DHEA rats in comparison with control group. Coadministration of HLF remarkably decreased the levels of all of these hormones, which was consistent with a reduction in follicular cysts. Similarly, HLF uptake alone showed no notable changes with respect to serous hormones. Our data demonstrate the significant upregulation of serous hormones in the DHEA-induced PCOS model. 


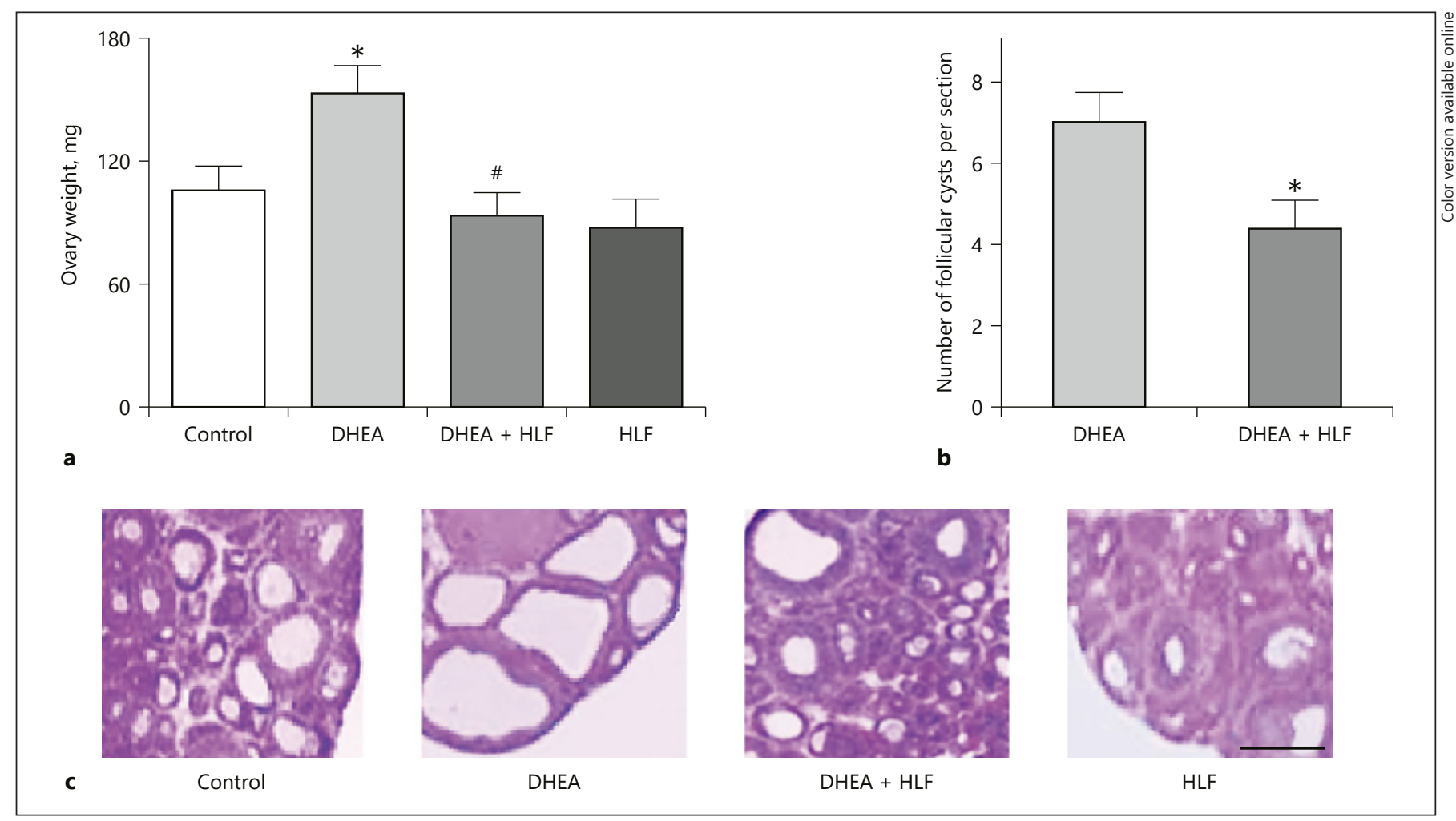

Fig. 3. Effect of HLF on the weight and morphology of the ovaries in rats. a Ovarian weight. b Number of follicular cysts. c Photos of ovaries in 4 groups. Scale bar, $50 \mu \mathrm{m}$. H\&E. ${ }^{*} p<0.05$ control versus DHEA group, ${ }^{\#} p<0.05$ DHEA versus DHEA + HLF group.

Table 1. Effect of HLF on the levels of serum hormones in rats

\begin{tabular}{lllccl}
\hline Group & LH $(\mathrm{IU} / \mathrm{L})$ & FSH $(\mathrm{IU} / \mathrm{L})$ & $\mathrm{E} 2(\mathrm{pg} / \mathrm{mL})$ & $\mathrm{P}(\mathrm{ng} / \mathrm{mL})$ & $\mathrm{T}(\mathrm{ng} / \mathrm{mL})$ \\
\hline Control $(n=8)$ & $21.60 \pm 2.48$ & $4.45 \pm 0.65$ & $5.7 \pm 0.18$ & $76.5 \pm 19.2$ & $0.18 \pm 0.05$ \\
DHEA $(n=8)$ & $33.94 \pm 2.07^{* *}$ & $7.35 \pm 0.72^{* *}$ & $88.9 \pm 25.1^{* *}$ & $198.1 \pm 31.3^{* *}$ & $0.29 \pm 0.02^{* *}$ \\
DHEA + HLF $(n=7)$ & $23.65 \pm 3.13^{\#}$ & $5.76 \pm 0.42^{\#}$ & $26.7 \pm 4.9^{\#}$ & $95.7 \pm 21.9^{\#}$ & $0.23 \pm 0.05^{\#}$ \\
HLF $(n=6)$ & $22.11 \pm 1.98$ & $5.55 \pm 0.36$ & $4.7 \pm 0.3$ & $58.3 \pm 21.1$ & $0.23 \pm 0.01$ \\
\hline
\end{tabular}

Values represent the mean \pm SEM. HLF, hawthorn leaf flavonoids; DHEA, dehydroepiandrosterone; LH, luteinizing hormone; FSH, follicle-stimulating hormone; E2, estradiol; $\mathrm{P}$, progesterone; T, testosterone. ${ }^{* *} p<$ 0.01 control vs. DHEA group, ${ }^{\#} p<0.05$ DHEA vs. DHEA + HLF group, one-way ANOVA, Tukey post hoc test.

Effect of HLF on IR and Estrous Cycle in Rats

IR status was assessed in PCOS rats in response to HLF administration. As shown in Figure 4a, IR, measured as the ratio between fasting insulin and glucose, was significantly induced in the DHEA-induced PCOS rats in comparison with controls $(3.62 \pm 0.21$ vs. $2.51 \pm 0.28, p<$ 0.01 ), and completely inhibited by simultaneous oral uptake of HLF. Administration of HLF alone showed no ef- fect on IR compared to controls. Similar results were obtained in the experiments involving postadministration of HLF ( $2.27 \pm 0.25$ in control vs. $2.74 \pm 0.17$ in DHEA + HLF, $p>0.05$, online supplementary Figure S1D).

We evaluated the estrous cycle as another index of ovarian function in PCOS rats upon HLF treatment. The stage of the estrous cycle was confirmed by vaginal smear. All rats from either control or HLF groups exhibited a 

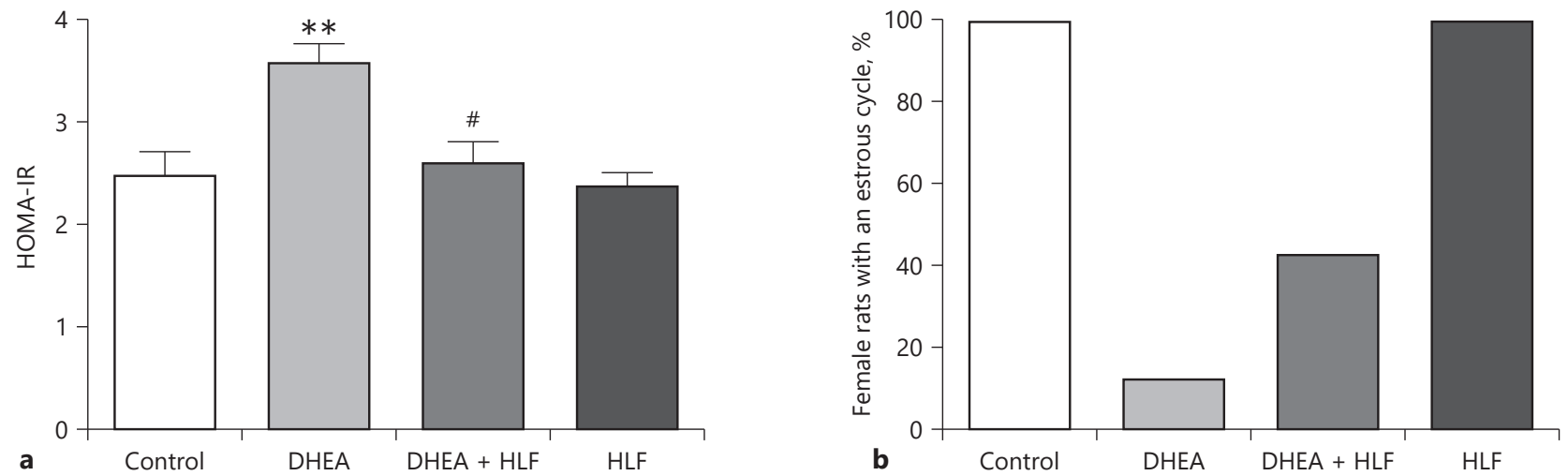

Fig. 4. Effect of HLF on the insulin resistance and estrous cycle in rats. a HOMA-IR of each group. b Percentage of rats exhibiting a complete estrous cycle. ${ }^{* *} p<0.01$ control versus DHEA group, ${ }^{\#} p<0.05$ DHEA versus DHEA + HLF group.

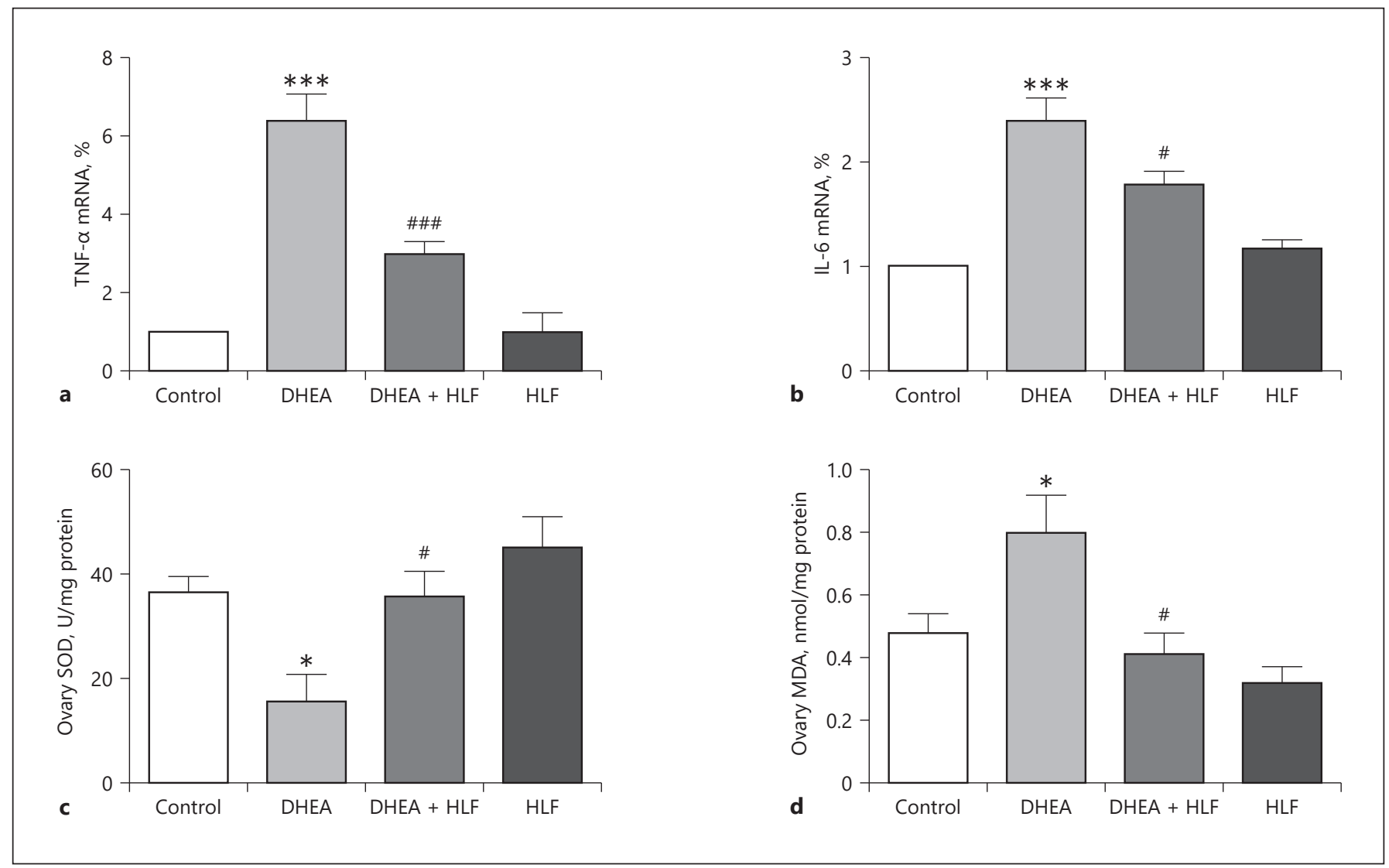

Fig. 5. Effect of HLF on the expression of inflammatory mediators and the oxidative stress in rats. a mRNA level of TNF- $\alpha .{ }^{* * *} p<0.001$ control versus DHEA group, ${ }^{\# \#} p<0.001$ DHEA versus DHEA + HLF group. b mRNA level of IL-6. ${ }^{* * *} p<0.001$ control versus DHEA group, ${ }^{\#} p<0.05$ DHEA versus DHEA + HLF group. c SOD in the ovaries. d MDA in the ovaries. ${ }^{*} p<0.05$ control versus DHEA group, ${ }^{*} p<0.05$ DHEA versus DHEA + HLF group. 
normal estrous cycle, while DHEA-induced PCOS rats had a significantly impaired cycle; only 1 of 8 rats in the DHEA group possessed a normal estrous cycle (Fig. 4b). Administration of HLF from the beginning restored the estrous cycle, and 3 of 7 DHEA rats displayed a normal estrous cycle, but this was not significant (online suppl. Table S1). Postadministration of HLF for 3 weeks caused significant recovery of the estrous cycle (online suppl. Table S2). Our data suggest that application of HLF alleviated IR and improved the dysfunction of the estrous cycle in the PCOS rats.

\section{Effect of HLF on the Expression of Inflammatory}

Mediators and Oxidative Stress in Rats

We then sought to elucidate the mechanism underlying the beneficial effect of HLF against PCOS in our DHEA-induced disease model. We examined levels of inflammatory mediators and oxidative stress in the blood collected from each group. As shown in Figure 5a, the relative expression of TNF- $\alpha$ was dramatically induced in DHEA rats with an average 6-fold increase in comparison with controls. Coadministration of HLF significantly suppressed the upregulation of TNF- $\alpha$ by DHEA. Consistent with previous observations, a single application of HLF had no influence on TNF- $\alpha$ expression. Serous IL-6 was also induced in DHEA rats and suppressed by the coadministration of HLF (Fig. 5b), indicating the evident inflammatory response in DHEA-treated animal.

Oxidative stress was measured by determining the relative SOD activity and MDA content. As shown in Figure $5 c$, the activity of SOD was greatly inhibited in the DHEA group in comparison with controls, and was subsequently induced by coadministration of HLF. The potentially direct effect of HLF on SOD activity in the ovaries was excluded as well. The end-product of lipid oxidation, MDA, was significantly increased in DHEA group and decreased by the application of HLF (Fig. 5d). Therefore, our results indicated high oxidative stress in DHEA-induced rats, which consequently contributes to the incidence of PCOS.

\section{Discussion}

In this study, we systematically investigated the potential benefits of HLF against PCOS in a rat model of disease. The s.c. injection of DHEA for 21 days successfully induced PCOS syndrome in SD rats. The success in establishing this disease model was experimentally confirmed in our study. The continuous monitoring of body weight revealed a significant induction of body and ovary weight gain in the PCOS group. Histological examination of ovarian sections showed characteristic features of PCOS in the DHEA-treated rats, including a swollen appearance, multiple dilated cystic follicles, atretic follicles, and well-developed theca interna cells. In addition, consistent with previous reports that DHEA induces a remarkable increase in luteinizing hormone, follicle-stimulating hormone, estradiol, progesterone, and testosterone in PCOS rats.

Further evaluation demonstrated that DHEA stimulated IR and inhibited the estrous cycle. Notably, oral administration of HLF partially abrogated gain of body/ ovarian weight in the PCOS group. Simultaneous uptake of HLF greatly suppressed the formation of follicular cysts. The serum hormones were almost completely restored to the normal conditions upon coadministration of HLF. The IR was completely abolished by HLF and the impaired estrous cycle in the PCOS rats was improved to some extent.

Regarding the mechanism involved, we provide evidence that DHEA significantly exacerbated the inflammatory milieu in the ovaries, indicated by the upregulation of both TNF- $\alpha$ and IL-6. The oxidative stress was also intensified by DHEA treatment as exemplified by decreased SOD activity and an increase in MDA (the endproduct of lipid oxidation).

Our results also provided evidence of anti-inflammatory and antioxidant activity, widely characterized in other diseases. Both inflammatory reactions and oxidative stress apparently and critically contribute to the etiology of PCOS in the DHEA-induced disease model, and were blocked significantly by the coadministration of HLF. We have highlighted, for the first time, the preventive application of HLF against PCOS. This warrants further clinical investigation. Notably, the detailed molecular mechanisms underlying the anti-inflammatory and antioxidant properties of HLF in the pathogenesis of PCOS are currently still elusive and need to be addressed in future investigations.

Hyperinsulinemia is a complication of PCOS, with excessive serous insulin and an association with IR [17]. Hyperinsulinemia consequently contributes to anovulation, folliculogenesis deficiency, and retarded follicular development. In this study, we investigated IR in PCOS model rats by measuring fasting blood insulin and glucose and calculating the HOMA-IR index. In line with previous reports [16], our data demonstrated stimulated IR in DHEA-treated ovaries which was then readily alleviated by oral administration of HLF. 
Serous estradiol increases during the formation of follicular cysts, estrous cycle dysfunction, altered ovarian steroidogenesis, and anovulation, and is causally linked to the hyperandrogenism in PCOS patients and the DHEA-induced disease animal model [18]. Sander et al. [19] demonstrated that metformin restored ovulation in PCOS and improved ovarian-related parameters in DHEA androgenized mice. Jang et al. [16] reported that the oriental medicine, Kyung-ok-ko, decreased the size and number of follicular cysts, restored estrous cycle arrest, and decreased elevated serum estradiol in PCOS rats. We assessed the effect of HLF against PCOS in the DHEAinduced disease model. Our results demonstrated that oral administration of HLF significantly alleviated the PCOS, inhibited follicular cyst formation, improved IR, and restored the estrous cycle. Our data suggest that HLF holds great promise for clinical application in PCOS.

\section{Conclusion}

PCOS is causally linked to chronic inflammation which is predominantly mediated by the macrophage cells [20]. Clinical investigation has identified that TNF- $\alpha$ and IL- 6 are elevated in nonobese/nondiabetic PCOS patients [21]. Meta-analysis has also uncovered the association of TNF- $\alpha, I L-6$, and $I L-1 \beta$ gene polymorphisms with polycystic ovary syndrome [22, 23]. Rezvanfar et al. [24] proposed dual targeting of TNF- $\alpha$ and free-radical toxic stress as a promising strategy to manage experimental PCOS. Puder et al. [25] demonstrated that women with PCOS were sensitive to the TNF- $\alpha$-lowering effect of glucose-induced hyperinsulinemia. The elevation of TNF- $\alpha$ and IL-6 mRNA expression has also been found in the visceral and s.c. adipose tissue of PCOS rats [26]. Consistent with these observations, we demonstrated here that DHEA treatment notably stimulated TNF- $\alpha$ and IL- 6 expression in the ovaries, while coadministration of HLF restored these cytokines.

In addition, accumulated clinical and experimental evidence suggests an association between oxidative stress and PCOS. For instance, Victor et al. [27] showed that IR in PCOS patients enhanced oxidative stress and leukocyte adhesion. Kocer et al. [28] demonstrated that metformin improved PCOS, probably by decreasing oxidative stress and improving IR. Consistent with this, we demonstrated that oral administration of HLF significantly suppressed the upregulation of both TNF- $\alpha$ and IL- 6 induced by DHEA, which might mechanistically contribute to its preventive effect against PCOS progression. Our study highlighted the potential therapeutic application of HLF in PCOS.

\section{Disclosure Statement}

The authors declare that they have no conflicts of interest.

\section{References}

1 Carmina E, Lobo RA. Polycystic ovary syndrome (PCOS): arguably the most common endocrinopathy is associated with significant morbidity in women. J Clin Endocrinol Metab. 1999 Jun;84(6):1897-9.

2 Goodarzi MO, Dumesic DA, Chazenbalk G, Azziz R. Polycystic ovary syndrome: etiology, pathogenesis and diagnosis. Nat Rev Endocrinol. 2011 Apr;7(4):219-31.

3 Teede H, Deeks A, Moran L. Polycystic ovary syndrome: a complex condition with psychological, reproductive and metabolic manifestations that impacts on health across the lifespan. BMC Med. 2010 Jun;8(1):41.

4 De Leo V, Musacchio MC, Cappelli V, Massaro MG, Morgante G, Petraglia F. Genetic, hormonal and metabolic aspects of PCOS: an update. Reprod Biol Endocrinol. 2016 Jul; 14(1):38.
5 Huang A, Brennan K, Azziz R. Prevalence of hyperandrogenemia in the polycystic ovary syndrome diagnosed by the National Institutes of Health 1990 criteria. Fertil Steril. 2010 Apr;93(6):1938-41.

6 Strauss JF 3rd. Some new thoughts on the pathophysiology and genetics of polycystic ovary syndrome. Ann N Y Acad Sci. 2003 Nov;997(1):42-8.

7 Mortada R, Williams T. Metabolic syndrome: polycystic ovary syndrome. FP Essent. 2015 Aug;435:30-42.

8 Sathyapalan T, Atkin SL. Mediators of inflammation in polycystic ovary syndrome in relation to adiposity. Mediators Inflamm. 2010; 2010:758656.

9 Legro RS, Arslanian SA, Ehrmann DA, Hoeger KM, Murad MH, Pasquali R, et al.; Endocrine Society. Diagnosis and treatment of polycystic ovary syndrome: an Endocrine Society clinical practice guideline. J Clin Endocrinol Metab. 2013 Dec;98(12):4565-92.
10 Moran LJ, Ko H, Misso M, Marsh K, Noakes $\mathrm{M}$, Talbot $\mathrm{M}$, et al. Dietary composition in the treatment of polycystic ovary syndrome: a systematic review to inform evidence-based guidelines. Hum Reprod Update. 2013 SepOct;19(5):432.

11 Lord JM, Flight IH, Norman RJ. Metformin in polycystic ovary syndrome: systematic review and meta-analysis. BMJ. 2003 Oct;327(7421): 951-3.

12 Legro RS, Zaino RJ, Demers LM, Kunselman AR, Gnatuk CL, Williams NI, Dodson WC. The effects of metformin and rosiglitazone, alone and in combination, on the ovary and endometrium in polycystic ovary syndrome. Am J Obstet Gynecol. 2007;196:402 e401-10.

13 Li Z, Xu J, Zheng P, Xing L, Shen H, Yang L, et al. Hawthorn leaf flavonoids alleviate nonalcoholic fatty liver disease by enhancing the adiponectin/AMPK pathway. Int J Clin Exp Med. 2015 Oct;8(10):17295-307. 
14 Fu JH, Zheng YQ, Li P, Li XZ, Shang XH, Liu JX. Hawthorn leaves flavonoids decreases inflammation related to acute myocardial ischemia/reperfusion in anesthetized dogs. Chin J Integr Med. 2013 Aug;19(8):582-8.

15 Min Q, Bai Y, Zhang Y, Yu W, Zhang M, Liu $\mathrm{D}$, et al. Hawthorn leaf flavonoids protect against diabetes-induced cardiomyopathy in rats via PKC- $\alpha$ signaling pathway. Evid Based Complement Alternat Med. 2017;2017: 2071952.

16 Jang M, Lee MJ, Lee JM, Bae CS, Kim SH, Ryu $\mathrm{JH}$, et al. Oriental medicine Kyung-Ok-Ko prevents and alleviates dehydroepiandrosterone-induced polycystic ovarian syndrome in rats. PLoS One. 2014 Feb;9(2):e87623.

17 Musso C, Shawker T, Cochran E, Javor ED, Young J, Gorden P. Clinical evidence that hyperinsulinaemia independent of gonadotropins stimulates ovarian growth. Clin Endocrinol (Oxf). 2005 Jul;63(1):73-8.

18 Diamanti-Kandarakis E, Christakou C, Kandarakis $\mathrm{H}$. Polycystic ovarian syndrome: the commonest cause of hyperandrogenemia in women as a risk factor for metabolic syndrome. Minerva Endocrinol. 2007 Mar;32(1): $35-47$.
19 Sander V, Luchetti CG, Solano ME, Elia E, Di Girolamo G, Gonzalez C, et al. Role of the $\mathrm{N}, \mathrm{N}^{\prime}$-dimethylbiguanide metformin in the treatment of female prepuberal $\mathrm{BALB} / \mathrm{c}$ mice hyperandrogenized with dehydroepiandrosterone. Reproduction. 2006 Mar;131(3):591602.

20 Benson S, Janssen OE, Hahn S, Tan S, Dietz $\mathrm{T}$, Mann K, et al. Obesity, depression, and chronic low-grade inflammation in women with polycystic ovary syndrome. Brain Behav Immun. 2008 Feb;22(2):177-84.

21 Amato G, Conte M, Mazziotti G, Lalli E, Vitolo G, Tucker AT, et al. Serum and follicular fluid cytokines in polycystic ovary syndrome during stimulated cycles. Obstet Gynecol. 2003 Jun;101(6):1177-82.

22 Guo R, Zheng Y, Yang J, Zheng N. Association of TNF-alpha, IL-6 and IL-1beta gene polymorphisms with polycystic ovary syndrome: a meta-analysis. BMC Genet. 2015 Jan; 16(1):5.

$23 \mathrm{Wu} \mathrm{H}$, Yu K, Yang Z. Associations between TNF- $\alpha$ and interleukin gene polymorphisms with polycystic ovary syndrome risk: a systematic review and meta-analysis. J Assist Reprod Genet. 2015 Apr;32(4):625-34.
24 Rezvanfar MA, Saeedi S, Mansoori P, Saadat S, Goosheh M, Shojaei Saadi HA, et al. Dual targeting of TNF- $\alpha$ and free radical toxic stress as a promising strategy to manage experimental polycystic ovary. Pharm Biol. 2016;54(1):80-90.

25 Puder JJ, Varga S, Nusbaumer CP, Zulewski H, Bilz S, Müller B, et al. Women with polycystic ovary syndrome are sensitive to the TNF-alpha-lowering effect of glucose-induced hyperinsulinaemia. Eur J Clin Invest. 2006 Dec;36(12):883-9.

26 Ghowsi M, Khazali H, Sisakhtnezhad S. Evaluation of TNF- $\alpha$ and IL- 6 mRNAs expressions in visceral and subcutaneous adipose tissues of polycystic ovarian rats and effects of resveratrol. Iran J Basic Med Sci. 2018 Feb; 21(2):165-74.

27 Victor VM, Rovira-Llopis S, Bañuls C, DiazMorales N, Martinez de Marañon A, RiosNavarro C, et al. Insulin resistance in PCOS patients enhances oxidative stress and leukocyte adhesion: role of myeloperoxidase. PLoS One. 2016 Mar;11(3):e0151960.

28 Kocer D, Bayram F, Diri H. The effects of metformin on endothelial dysfunction, lipid metabolism and oxidative stress in women with polycystic ovary syndrome. Gynecol Endocrinol. 2014 May;30(5):367-71. 